\title{
ARTICLE
}

\section{Tracing past population migrations: genealogy of steroid 21-hydroxylase (CYP21) gene mutations in Finland}

\author{
A ntti L evo ${ }^{1}$, Jarmo J ääskeläinen ${ }^{2}$, Pertti Sistonen ${ }^{1}$, M arja-K aisa Sirén ${ }^{1}$, \\ $\mathrm{R}$ aimo Voutilainen ${ }^{2}$ and Jukka Partanen ${ }^{1}$ \\ ${ }^{1}$ Finnish Red Cross Blood Transfusion Service, Tissue Typing L aboratory, $\mathrm{H}$ elsinki \\ ${ }^{2}$ K uopio U niversity H ospital, D epartment of Pediatrics, Kuopio, Finland
}

\begin{abstract}
The genealogic origin of steroid 21-hydroxylase gene (CYP21) mutations and associated haplotypes was determined in $\mathbf{7 4}$ unrelated Finnish families with CY P 21 deficiency (congenital adrenal hyperplasia, CA H). These families account for two thirds (85/119) of all diagnosed patients of Finnish descent found in this country. We recently demonstrated that multiple founder mutations each associated with a particular haplotype can be found in Finland. Interestingly, some of the haplotypes were identical to those observed in various $E$ uropean populations, whereas others have not been described elsewhere, indicating a local and perhaps a more recent origin. In the present report we show that each of the major founder haplotypes originates from a particular geographic region of Finland. Thus many local genetic isolates are to be expected in Finland. 0 ur finding is in a clear contrast to the genetic diseases known as the 'Finnish disease heritage', in which only one mutation usually predominates. Some of the CY P21 haplotypes proved very informative for analysis of the history of the Finnish population. For example, the origin of one frequent haplotype was shown to cluster in a region assumed by archaeological data to be a major site of immigration by settlers of either Scandinavian or B altic origin during the first centuries A D. A s this haplotype is frequent in many E uropean patient populations, we provide independent genetic evidence of this Iron A ge immigration. On the other hand, another frequent haplotype found solely in Finland reflects a more recent (post 15th century) settlement expansion. Consequently, well characterised and sufficiently frequent autosomal gene markers can provide useful information on migrations both between and within populations.
\end{abstract}

Keywords: steroid 21-hydroxylase; CYP21; congenital adrenal hyperplasia; major histocompatibility complex; gene mutations; haplotype markers; population migrations; Finnish population history

\section{Introduction}

The special features of the Finnish population structure ${ }^{1-4}$ provide a good opportunity for studying the origin and distribution of genetic traits. This attribute has proven particularly advantageous in the characterisation of inherited diseases known as the 'Finnish

Correspondence: A ntti L evo or J ukka Partanen, FR C B lood Transfusion Service, Tissue Typing L aboratory, K ivihaantie 7 , FIN-00310 H elsinki, Finland. Tel: +358 9 58011; Fax: +358 9 $5801 \quad 429 ; \quad$ E-mail: antti.levo@bts.redcross.fi, or jukka.partanen@bts.redcross.fi

R eceived 20 January 1998; revised 13 A ugust 1998; accepted 19 A ugust 1998 disease heritage'. This concept embodies over 30 mostly autosomal recessive diseases that are more prevalent in Finland than elsewhere. ${ }^{3-5} \mathrm{U}$ sually a single mutation enriched as a result of founder effect accounts for most, if not all, cases of defective alleles. Only in a few other inherited diseases has the mutation spectrum in Finland been analysed to same extent. Classic congenital adrenal hyperplasia $(\mathrm{CAH})$ due to steroid 21-hydroxylase (CY P21) deficiency. ${ }^{6,7}$ shows neither an increased nor a decreased incidence in Finland, but one ( 1 in 15000 newborns) consistent with that reported worldwide. $^{8,9}$ In clear contrast to the diseases of the Finnish disease heritage, multiple independent founder mutations each associated with a particular haplotype are 
found in $\mathrm{CAH}$ in Finland. ${ }^{10}$ Therefore, $\mathrm{CAH}$ could serve as a model for investigating the genetic features underlying a 'classic' inherited disease in Finland. It should be of interest to compare these results to those obtained from analyses of the 'Finnish disease heritage'. In addition, as some - but not all - of the founder mutation-haplotypes we observed in Finland ${ }^{10}$ are also found in other E uropean populations, and as the carrier frequency of the CY P21 defect is sufficiently high $(\sim 1 / 60)$, CY P21 mutations could serve as informative markers for the evaluation of genetic relationships between populations. $D$ ata obtained from analyses of mitochondrial and Y-chromosomal variation (eg $L$ ahermo et $\mathrm{al}^{11}$ ) seem most useful in demonstrating more ancient relationships, whereas certain autosomal markers could provide information of more recent periods.

The gene encoding the steroid 21-hydroxylase enzyme, CY P21, as well as a closely related pseudogene CY P21P, ${ }^{12,13}$ are tandemly located in the human major histocompatibility complex (M HC) on chromosome $6 p 21.3$ (Figure 1). With the exception of a few novel sporadic point mutations reported, only two types of mutation are found in CY P21 deficiency: ${ }^{14}$ large deletions leading to a nonfunctional CY P21P/CY P21 fusion gene, and gene conversion type of mutation where a part of the functional gene has been replaced by a pseudogene-derived deleterious segment. A s the pseudogene carries only a limited number of mutations, ${ }^{14}$ identical but obviously independently borne mutations in the CY P21 gene have been detected in all populations studied so far. Thus, haplotype information is essential for analysis of origins of CY P21 mutations. We now present mutation-haplotype data, as well as genealogic data, of more than two thirds of all patients found in Finland. This allowed us to trace the genea-

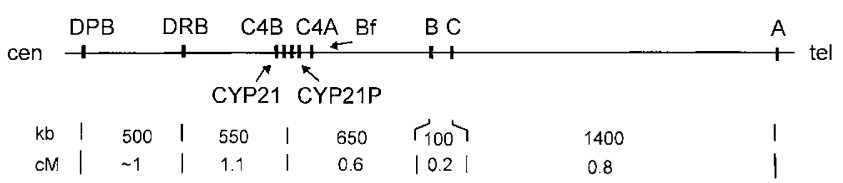

Figure $1 \mathrm{H}$ aplotype marker loci in the human M HC region.

Schematic organisation of the polymorphic marker loci in the human major histocompatibility complex $(\mathrm{M} \mathrm{HC})$ on chromosome $6 p 21.3$ used for haplotype construction; centromere to the left, telomere to the right. Their approximate physical and genetic distances are taken from references. ${ }^{17,29}$ The functional steroid 21-hydroxylase gene (CYP21) and 21-hydroxylase pseudogene (CY P21P) are duplicated in tandem with the complement $C 4$ genes $(C 4 A$ and $C 4 B) . k b=$ kilobase, $\mathrm{CM}=$ centi $\mathrm{M}$ organ, $\mathrm{Bf}=$ complement factor $\mathrm{B}$ gene. logic origin and geographical distribution of the multiple CY P21 mutation-haplotype combinations found in Finland.

\section{Materials and Methods}

\section{Patients}

Seventy-four unrelated Finnish families with $\mathrm{CAH}$ (85 patients) were analysed. 148 unrelated chromosomes plus two: in one family, both the mother and son were affected. In another family, due to a de novo mutation both maternal alleles were considered affected. The CY P21 mutations and clinical phenotypes of 78 of them have been reported previously. $^{8}$ In terms of sex ratio, clinical spectrum, and regional distribution, the patients analysed here are a representative sample of the total Finnish patient population. In our recent screening, altogether 120 patients with $\mathrm{CA} \mathrm{H}$ due to steroid 21-hydroxylase deficiency could be identified in Finland, resulting in an incidence of 1 in 15000 live births. $^{8}$

\section{CYP21 Genotypes}

G enotyping of disease-causing mutations in the CY P 21 gene as well as detailed molecular analysis of the most common affected haplotypes, ie mapping of deletion and conversion breakpoints in CY P 21, was performed by using allele-specific $P C R$, direct DNA sequencing, PCR -R FL P, standard genomic RFLP followed by Southern blotting, and pulsed-field gel electrophoresis (PFGE) as described in detail previously. $8,10,15,16$

\section{Haplotype Markers}

Seven polymorphic markers (HLA -A , -B, -Cw, -D R B 1, C 4A, $\mathrm{C} 4 \mathrm{~B}$ and $\mathrm{Bf}$ ), in the human $\mathrm{MHC}$ region ${ }^{17}$ encompassing approximately $2.7 \mathrm{Mbp}$ of DNA flanking the CYP21 gene were typed (Figure 1). In some cases, HLA-DPB 1 typings were also performed, expanding the typed genomic region to approximately $3.2 \mathrm{M} \mathrm{bp}$.

In addition to standard HLA typing methods, ${ }^{10,16}$ we utilised a PCR-SSP 'low-resolution' kit (Dynal AS, O slo, Norway) for the determination of the $H L A-A,-B$ and $-C w$ alleles of 18 patients not previously typed. A Iso, all samples serologically typed as HLA-B40 were retyped at the DNA level. A ccordingly, the haplotype marked as B 48 S01 D R 8 in the present study refers to haplotype B 40 S01 DR 8 of our previous reports. ${ }^{10,15,16}$ Some further samples were retyped to resolve serological ambiguities.

\section{Mutation-haplotype Combination Construction}

The CYP21 mutations and haplotypes in 51 of the 74 unrelated patients have been reported previously in detail. ${ }^{10}$ In 38 families both parents, and in six families one parent, were available for segregation analysis and hence accurate mutation-haplotype combination could be determined. Four additional samples were homozygous for the haplotype markers and CY P21 mutations. For the remaining 26 patients, the criteria for the haplotype reconstruction were as follows: a previously described mutation-haplotype combination ${ }^{10}$ was assumed if, firstly, an identical CY P21 mutation was observed, and, secondly, the sample had the characteristic alleles in a minimum of five loci (ie $H L A-B, B f, C 4 A, C 4 B$, and DRB 1) flanking the CYP 21 gene. These criteria, though 
arbitrary, were considered to give a reliable outcome, since each of the frequent haplotypes carried at least one marker that is rare in the general population. M ore allelic variation was allowed in certain families if informative members were available for segregation analysis. A Iso, two patients were included, for whom only the haplotype results were available, but no DNA for CYP21 mutation determination. In both cases, however, DNA samples of both parents could be analysed, thus allowing an accurate definition of their mutation-haplotype combinations.

\section{Genealogic Analyses}

A ncestors of patients were traced initially by questionnaires sent to all families enquiring the full names, dates, and places of birth of the patients' parents and grandparents. A $n$ answer was received from 49 families $(66.2 \%)$. In other cases, information on the pedigrees was obtained from public registers. Distant ancestors were identified using church parish registries, which provide nationwide information on births, deaths, and marriages extending typically some 300 years back (about 12-15 generations). To some extent, the data are accessible also on the World Wide Web (http:/ /www.genealogia.org/historia/fi). However, any information obtained was confirmed by local church registries. In general, the genealogy was traced back at least three generations, but even up to 13 generations in some families.

To investigate the geographic distribution of a mutation, the birthplaces of grandparents of patients carrying a particular mutation-haplotype combination were dotted on a map of Finland of the beginning of the present century, before two recent major population admixtures took place in the country - the resettlement of some 430000 ( >10\% of the population) $\mathrm{K}$ arelian evacuees in 1939-44, and the migration from rural regions to urban areas since the second world war.

Haplotype Markers in the Present General Population $\mathrm{HLA}$ antigen frequencies in Finland, as well as their regional variation, were recently reported by Sirén et al. ${ }^{18}$ The data were obtained from 10000 voluntary Finnish donors registered with the Finnish B one M arrow Donor R egistry.

\section{Results}

Seventy-four unrelated Finnish families with $\mathrm{CA} \mathrm{H}$ due to CY P21 deficiency (150 unrelated affected chromosomes) were analysed. They accounted for $71 \%$ (85/119) of all diagnosed patients of Finnish descent found in this country, ie all patients from whom blood samples for genotyping could be obtained. Nineteen apparently independent founder mutation-haplotypes totalling $83 \%(124 / 150)$ of all affected chromosomes could be identified. The individual frequencies and the associated markers of the four most frequent haplotypes are shown in Figure 2. Essentially, the haplotypes and their frequencies are identical to those recently reported. ${ }^{10}$ The remaining fifth of the chromosomes occurred in single families only. Their overall geo- graphic distribution in comparison to the summary of the founder haplotypes is presented in Figure $3 \mathrm{~A}$.

\section{Geographic Origin of Haplotypes}

No obvious close consanguinity was initially observed between the parents or grandparents of the patients. Figure $2 \mathrm{~A}-\mathrm{C}$ shows the birthplaces of the grandparents of carriers of the three most frequent mutationhaplotype combinations observed. E ach was found in more than 10 unrelated families. Together, these haplotypes accounted for nearly half $(44.7 \% ; 67 / 150)$ of all the affected chromosomes. $M$ ap $D$ in Figure 2 shows an example of the origin of one of the 16 lower-frequency haplotypes (2-6 unrelated families for a given haplotype; the 15 other maps are available from the authors on request). In all cases, the average date of birth of the grandparents was well before the second world war, that is before the major population movements in Finland.

It is evident that the three high-frequency haplotypes all have a rather limited geographic origin. The most prevalent haplotype (Figure 2A) shows three clear clusters. The most abundant is around the coastal town of Oulu in north-western Finland (Figure 2). A nother, closely connected to the Oulu cluster, is situated just 100 kilometres to the south of it and extends along the $\mathrm{K}$ alajoki river valley to the inland region of Savo. The third cluster is near the coastal towns in the VakkaSuomi region in south-western Finland (Figure2). Interestingly, the families of Vakka-Suomi have a recombinant haplotype carrying a different telomeric segment (A 2 Cw2 B 61 instead of A 24 Cw8 B 48). The origin of the ancestors of families carrying the second most common haplotype (Figure 2B) forms a continuum extending along the Kokemäki river from the coastal areas of Satakunta eastwards via $\mathrm{H}$ äme to Savo and $\mathrm{K}$ arelia in eastern Finland. The birthplaces in Figure $2 \mathrm{C}$ are more scattered, but it should be noted that they actually represent four distinct recombinant haplotypes, all demonstrated to have originated from the A 3 Cw6 B 47 F 10 DR 7 haplotype. ${ }^{19}$ By studying each recombinant separately, clear clustering can be observed. For example, those with B 18 follow the route of the Lestijoki river (Figure 2C).

The majority of haplotypes with a lower frequency also showed strong clusters in their origins. For example, the fourth most frequent haplotype (Figure 2D) has a strikingly restricted origin in the region of Southern O strobothnia. Nevertheless, some of the lowest frequency haplotypes (maps not shown) may actually represent separate mutational events. 


\section{Pedigrees and Consanguinity}

In general, there was remarkably little close consanguinity within the Finnish $\mathrm{CAH}$ families. Only two marriages between first cousins were found in the 74 pedigrees analysed. However, the parents of the patients were often found to share distant ancestors. Figure $4 \mathrm{~A}$ represents the family tree of one patient homozygous for the haplotype B 7 S31 DR 15 (I172N), whose pedigree includes multiple remote consanguineous marriages. Figure $4 \mathrm{~B}$ presents a case of shared ancestors, dating back to the late 1700s, of two additional patients with the same haplotype.

Some additional patients, carrying identical mutation-haplotype combinations, were demonstrated to be remotely consanguineous. A good example of a complex pedigree is depicted in Figure $4 C$, which shows the joint family tree of four unrelated patients, one of whom was homozygous, who all carried the most frequent haplotype (Figure 2A) They were found to have common ancestors, born in the region of the lijoki and $\mathrm{H}$ aukipudas parishes (located in the region of Oulu, Figure 2A ) in the late 1600s, and the lineages merge on multiple occasions. In other words, there are several possible lines of shared descent.

A common ancestor was also found for certain of the more rare haplotypes; for example, for the only two patients with a B17 S02 DR 13 (Q318X) haplotype (Figure 4D; map not shown). Finally, ancestors could often be traced back to a single parish and to the same or adjacent villages. O ccasionally, they even lived on the same farm, but no actual link demonstrating direct consanguinity was readily discoverable. This was the case, for example, in haplotypes B 40 S30 DR 1 (delA) and B 47 F 10 D R 7 (delB).

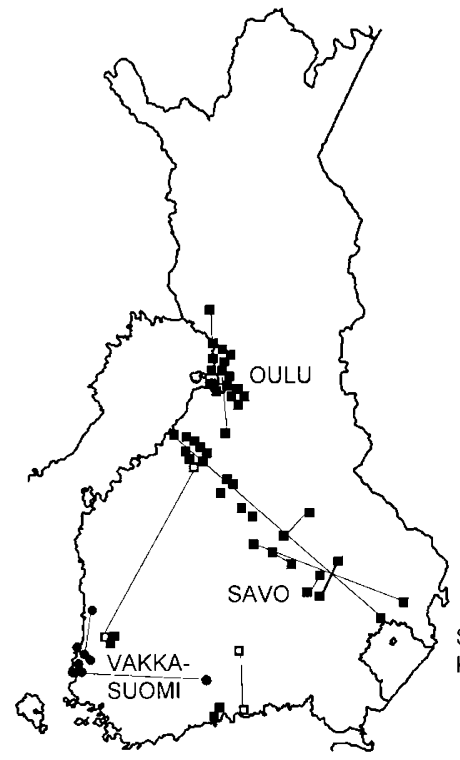

A24 Cw8 B48 S01 DR8
I172N
$19.3 \%(29 / 150)$
B.

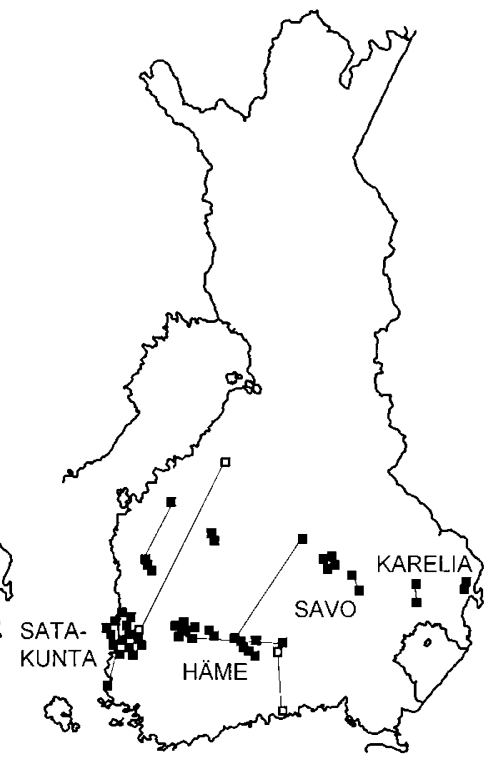

(A3) Cw3 B60(40) S30 DR1 $30 \mathrm{~kb}$ delA * $16.7 \%(25 / 150)$ c.
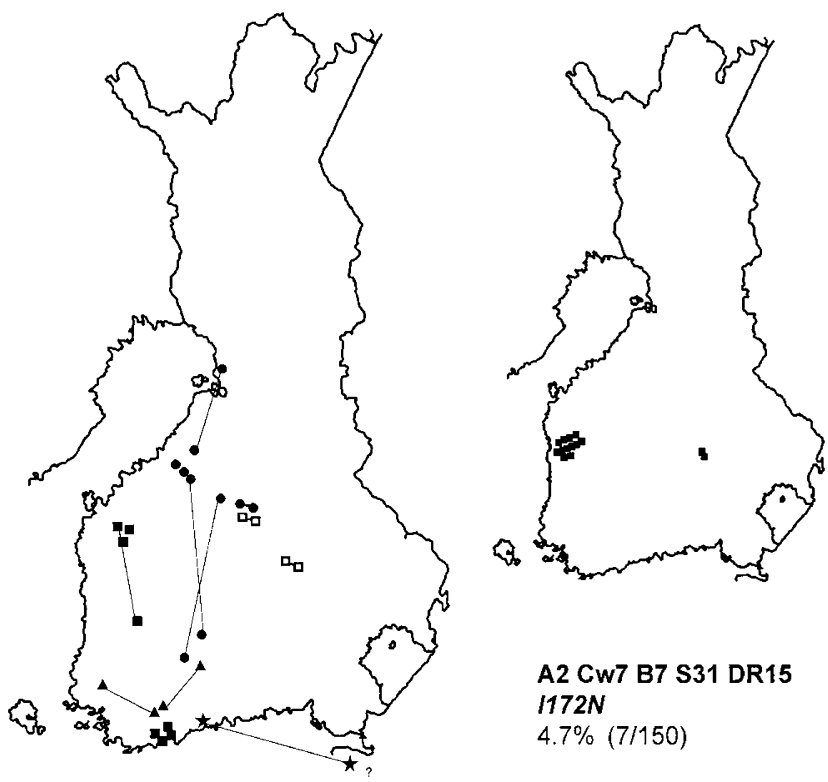

(A3 Cw6 B47) F10 DR7

$30 \mathrm{~kb} \mathrm{delB^{* }}$

$8.7 \%(13 / 150)$
D.

Figure 2A -D D istribution of the four common CYP21 mutations in Finland. Geographic distribution of the four most frequent CYP21-deficient haplotypes as shown by the birthplaces of grandparents of patients. The mutation and haplotype, as well as the observed frequency in patients, are given below each map. The maps show the territory of Finland in the beginning of the present century, consistent with the grandparents' mean year of birth (1912 in A; 1914 in B; 1915 in C; 1898 in D ). A s the grandparents were not available for genetic analyses, the birthplaces of both grandparents either on the maternal or the paternal side are shown. $\mathrm{H}$ ence, it should be noted that in fact only half of the points presented are true.

$M$ arried couples are connected with lines. In Figures $2 A$ and $C$, the recombinants were included in the same map and are marked by different symbols. In Figure 2A, indicates the A 2 C w2 B 61 haplotype instead of A 24 C w8 B 48. In Figure 2C: (A 3 C w7 B 18), $\boldsymbol{\Delta}(\mathrm{A} 3 \mathrm{C}$ w3 B 62), $\star$ (A 11 Cw4 B 35); the original A 3 Cw6 B 47. O pen symbols for all four grandparents indicate that the parents could not be studied. * delA and delB denote distinct large deletions with different breakpoints in the CY P 21 gene. ${ }^{10}$ 


\section{Markers for Disease Haplotypes in the Present Population}

We utilised the databank of the Finnish B one Marrow Donor Registry ( $n=10000$ individuals) to check whether any trace of the clusters could be seen in the present Finnish population. We selected only three haplotypes for this study, since we reasoned that in order to be able to interpret the results the haplotypes tested ought to have a readily identifiable tag, ie a rare HLA allele. We looked for the current place of residence of those individuals positive for $H L A \quad B 48$ together with $A 24$ and DR8, the haplotype in Figure $2 A$; for $B 47$ with $D R 7$, the haplotype in Figure $2 C$; and for alleles B 22 with DR 4 (the haplotype B 22 S45 DR 4 with mutation I172N + P453S, map not shown). No definitive conclusions could be drawn from the B 22 or B 47 searches. $O \mathrm{n}$ the other hand, the clear majority of B 48-positive haplotypes was found to occur also today in the same general region (data not shown), whence the CY P21-defective A 24 Cw8 B 48 S01 DR 8 haplotype originated (Figure $2 \mathrm{~A}$ ).

A.

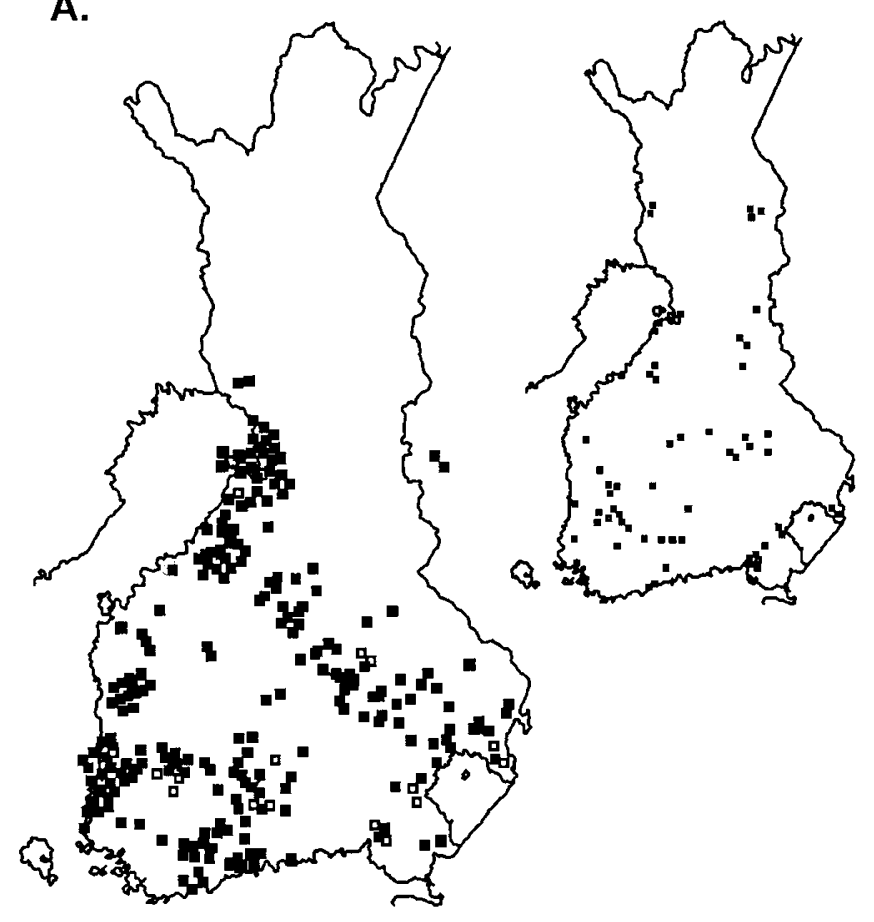

\section{Discussion}

The two major findings of the present study are that each of the frequent mutation-haplotype combinations has a clear restricted geographic origin in Finland, and that some of the haplotypes proved very informative for studies of the population history of the country. To our knowledge, this report is the first attempt to trace the origin of CY P21-deficient chromosomes in a human population.

$M$ any single-gene defects in Finland, particularly those belonging to the 'Finnish disease heritage', have been shown to be due to just one major mutation. ${ }^{3,4}$ CY P21 deficiency appears to be an exception: 19 founder mutations and a number of sporadic mutations exist in Finland ( $\mathrm{Levo}^{10}$ and the present report). A $\mathrm{n}$ explanation for this heterogeneity could be the high frequency of CY P21 deficiency. The worldwide carrier frequency has been estimated to be about $1 / 60,{ }^{9}$ from which Finland seems not to deviate. ${ }^{8}$ A ssuming that there has been no substantial change in the carrier rate

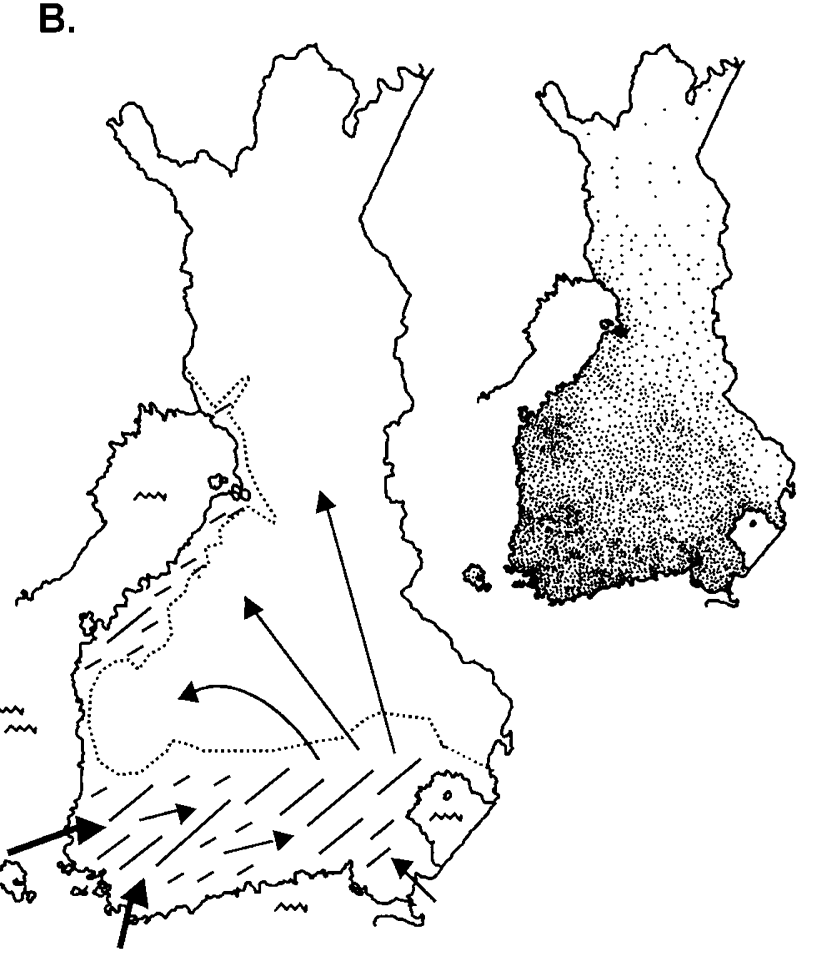

Figure 3 Overall distribution of CYP21 mutations in Finland, main lines of settlement and distribution of population. A G eographic distribution of birthplaces of ancestors of all analysed Finnish patients with CY P 21 defect carrying any one of the 19 founder haplotypes (left) as compared with the distribution of haplotypes occurring only in single families (right). B A schematic picture of the major population migrations in Finland (left); the arrows denote the main directions. Since the end of the glacial period (c. 9000 years ago) there has been gradual but continuous migration to Finland mainly from the south and southwest. The ruled area indicates permanent settlements before $1500 \mathrm{AD}$. O n the right: distribution of rural population in Finland at the turn of the century (1908); each dot represents 1000 inhabitants. A dapted from references. ${ }^{1,22-24}$ 
A.

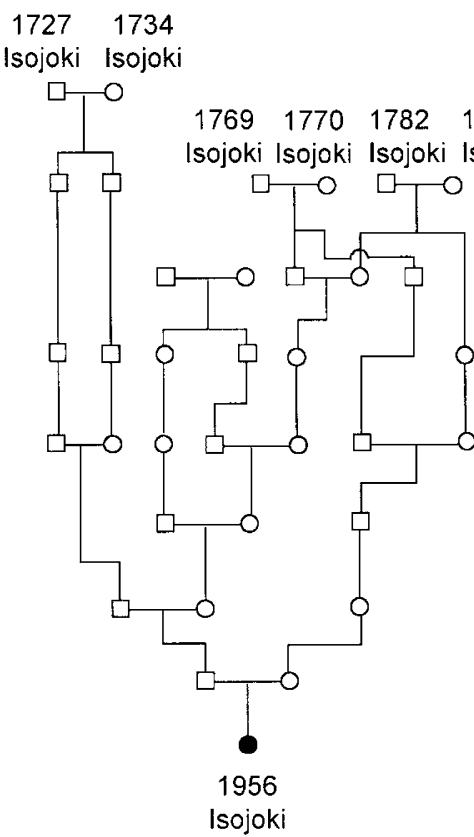

B.

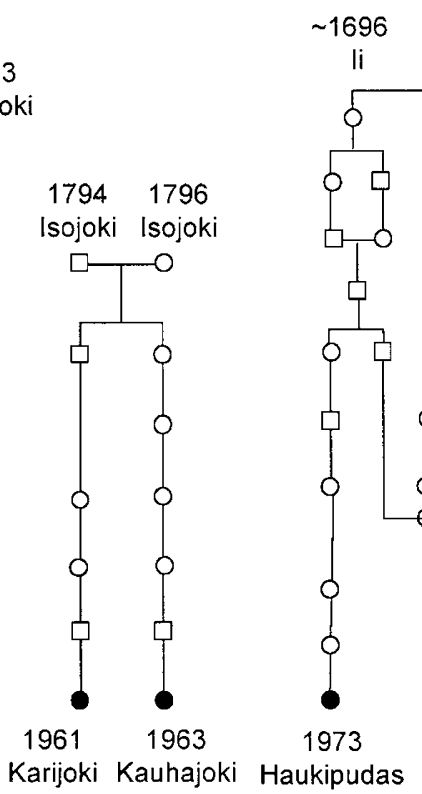

c.

D.

Figure 4 Typical pedigrees of Finnish CAH families A The ancestry of one patient homozygous for the B7 S31 DR 15 (I 172N) mutation showing multiple consanguinity. B A pedigree demonstrating direct consanguinity between two carriers of the B7 S31 DR 15 (I172N) mutation. C A joint family tree of four patients (one homozygous) carrying the B 48 S01 D R 8 (I 172N) mutation. The tree demonstrates common ancestors and how the lineages merge on multiple occasions. D A pedigree demonstrating direct consanguinity between the two carriers of the B 17 S02 D R 13 (Q 318X) mutation.

in the past, many of the early settlers of Finland may have carried a defective CY P21 gene. In addition, the frequency of de novo mutations in CY P21 is high (1-2\% of affected alleles), ${ }^{14}$ increasing further the mutational spectrum. $\mathrm{O}$ ur finding of genetic heterogeneity in CY P21 deficiency is of general importance for genetic studies of common disorders. The susceptibility loci for these diseases are usually thought to be frequent polymorphisms or mutations, rather than rare defective alleles. The genetic heterogeneity of these loci in the general Finnish population can similarly be expected to be rather high. However, in local subpopulations or clearly defined clinical subgroups one can still expect founder mutations. Indeed, recent studies of the genetics of asthma ${ }^{20}$ and multiple sclerosis ${ }^{21}$ in Finland have focused on local isolates. If we consider each founder CY P21 mutation separately, many of them in fact are very similar to the findings of the Finnish disease heritage. ${ }^{2-5}$ The overall geographic distribution of the CY P21 founder mutations in Finland is somewhat scattered (Figure $3 \mathrm{~A}$ left), demonstrating that there is no one particular area from which the majority of cases originate. This fits with the idea of a multiple founder effect.

U nexpectedly, we found evidence that even today some of the founder haplotypes are still mainly restricted to the same region where they are thought to have originated; the majority of individuals positive for the A 24, B 48, and DR 8 alleles live in the very same region as shown in Figure $2 A$. These findings are consistent with the concept of 'isolation by population density', introduced by Nevanlinna a quarter of a century ago $^{1}$ to interpret the deviations in gene frequencies of local subpopulations. Thus the population admixture in the more rural areas is still low and some present-day subpopulations are good representations of the genetic features of the population which occupied the region in the past.

A Ithough a clear geographic origin could be inferred for many of the mutations, a common ancestor was identified only rarely. However, it should be noted that in most cases we actually did not even attempt to trace back all relatives; our aim was merely to show evidence of shared origin. In addition, many shared lines that 
indicate multiple consanguinity were evident in more complex pedigrees, like the one shown in Figure $4 \mathrm{C}$. Therefore it cannot be taken for granted that any of them actually represents the shared origin of this particular gene mutation. A lthough inbreeding in Finland has been thought to be low, for example marriages between first cousins were prohibited by law until 1872, the remote consanguinity found in the pedigrees may be a common feature in rural areas of Finland. This effectively hampers definitive identification of a shared ancestor based on pedigrees only.

The genealogic and geographic data collected in the present paper are interesting in connection with the known population history of Finland. ${ }^{1,22-24}$ A rchaeological evidence clearly indicates that Finland has been inhabitated since the end of the glacial period some 9000 years ago, but these earliest residents presumably have only a minor influence on today's gene pool. There has been gradual, rather low in number but continuous, migration to Finland, mainly from the south and southwest across the G ulf of Finland and the B altic Sea throughout prehistoric times (Figure 3B). The total number of these settlers, who were predominantly of Baltic, Germanic, and Scandinavian origin must have been small, tens at a time rather than thousands. Additional immigration to Finland has been sparse throughout historical times. O nly the southwestern and southern coastal parts of Finland were inhabited rapidly and early (Figure $3 \mathrm{~B}$ ). The vast northern and northwestern wildernesses were settled permanently as late as in the 16th and 17th centuries, only 15-20 generations ago, mainly by a relatively small number of families from the Savo region.

The distribution of the two most frequent CY P21deficient haplotypes (Figures 2A and Figure 2B) is notably consistent with two major population migrations documented in Finland (Figure 3B). The geographic distribution of ancestors of the second most common haplotype, B 40 S30 D R 1 with a large deletion (Figure 2B), presents a clear continuum in the southwestern region stretching from west to east. The southwestern region is considered an area of the most ancient inhabitation., ${ }^{1,22,23}$ This pattern parallels an archaeologically well documented population movement in Finland. ${ }^{22}$ There has been a staged spread of permanent inhabitation (beginning in the I ron A ge in approximately 200 A D ) from the southwestern coastal regions along the Kokemäenjoki river valley to $\mathrm{H}$ äme (about 600-700 A D) extending gradually to the eastern parts of Finland (about 800 A D). M oreover, archaeo- logical findings in these areas are continuous and similar to those found in southern Scandinavia and in the areas east of the B altic Sea. ${ }^{1,22}$ A s the identical B 40 S30 DR 1 haplotype is also consistently found in patients in many other populations in Europe, ${ }^{10}$ for example its frequency in Irish patients is $26 \%,{ }^{25}$ this mutation was probably introduced into the Finnish population by immigration and began to spread nearly 2000 years ago. The fact that we found no consanguinity between any families is in line with this haplotype's believed ancient origin. We have also analysed one patient of E stonian ancestry (unpublished results), who was homozygous for this very same haplotype. U nfortunately there are no haplotype data on Swedish or other Scandinavian patients, so we are not able to make a better guess of its origin.

On the other hand, the geographic distribution of the most frequent haplotype, B 48 S01 D R 8, which seems to be restricted to Finland, ${ }^{10}$ is in agreement with another major population migration within the country. The expansion of people from Savo from the late 15th century onward is well documented. ${ }^{24}$ It started in southern Savo with one of its main streams proceeding rapidly northwestward, including the $K$ alajoki river and Oulu areas (Figure 3B). Indeed, based on parish records, it is known that the first permanent settlers in the upper reaches of the $K$ alajoki river in the early 1500s came from Savo. ${ }^{26}$ Consequently, we can speculate that this particular mutation spread into the population at least some $400-500$ years ago. Thereafter it was enriched by random drift in the various isolated rural subpopulations. This interpretation fits with our genealogic analyses, which demonstrate that the family lineages merge several times, their most recent common ancestors dating from the 17th century. However, the observation of a third cluster in the old southwestern settlement area implies that this haplotype with $A 2$ Cw2 B 61 alleles may actually represent the 'original' mutated haplotype possibly introduced into the Finnish population by immigration at a much earlier point in time, whereas the B 48 chromosome might be a recombinant that has become greatly enriched over the centuries in the more recently inhabited northern regions. Yet another interpretation is that the two haplotypes are actually independent.

The origin of the third most common haplotype, B 47 F 10 D R 7 carrying a large deletion, is more obscure due to the lower number of cases. It also shows more haplotypic variation. The scattered geographic distribution of birthplaces (Figure $2 \mathrm{C}$ ) suggests that it may have 
more than one single origin. It should be noted that this deletion haplotype is widespread in most patient groups of E uropean descent. ${ }^{10}$ Interestingly, the overall distribution of the B 47 haplotypes shows similarity with the clustering of the cystic fibrosis mutation $(\Delta) F 508$ in Finland, ${ }^{27}$ another widespread and ancient human mutation.

The number of individuals carrying one of the lower frequency haplotypes was small, only 2-6 families per haplotype, but they also showed tight geographic clustering. For example, the fourth most frequent haplotype (Figure 2D) has a strikingly narrow origin. U nlike the three most common defective haplotypes, whose $\mathrm{HLA}$ allele combinations are extremely rare in the general Finnish population, the B7 S31 DR 15 combination has a frequency of $6 \%$ in Finland, ${ }^{28}$ making it one of the most frequent haplotypes. $M$ any facts strongly suggest a common and fairly recent origin for this mutation: firstly, virtually no allelic variation was seen in the patients' affected haplotypes; secondly, two of the five patients were homozygous for it; and finally, all but one patient's ancestors map to three neighbouring parishes (Figure 2D). The most recent common ancestors of two of the patients with this haplotype were born in the late 18th century (Figure $4 B$ ). We can therefore speculate that the mutation occurred at least 200 years ago. A few possible exceptions for the limited origin were also observed, for instance, the haplotypes B7 S31 DR 13 and B62 S42 DR 4 (maps not shown), both with the 12 splice mutation.

In summary, the CYP21 mutations in Finland appeared very disparate in age and origin. We were able to provide independent, though speculative, genetic evidence of the two major population migrations that previously have been documented by archaeological and historical findings. Consequently, well characterised and sufficiently frequent autosomal gene markers can provide useful information on migrations both between and within populations. The data from various autosomal, mitochondrial and $Y$-chromosomal markers may each enlighten different perspectives of human population histories.

\section{Acknowledgements}

We are grateful to all the Finnish $\mathrm{CA} \mathrm{H}$ families who kindly provided family data for this study. We also want to express our sincere gratitude to Dr Matti Huurre, Dr Markku $M$ äkivuoti, and Professor Jouko Vahtola for their expert advice in the fascinating fields of archaeology and history. We also thank the personnel of church registry offices throughout the country for kindly contributing genealogic data. This work was financially supported by the Foundation for Pediatric R esearch, the Paulo Foundation, the Instrumentarium Scientific Fund, the Maud Kuistila Memorial Foundation, the Jalmari and Rauha A hokas Foundation, and the Finnish Cultural Foundation.

\section{References}

1 Nevanlinna HR: The Finnish population structure. A genetic and genealogic study. Hereditas 1972; 71: 195-236.

2 Norio R, Nevanlinna HR, Perheentupa J: Hereditary diseases in Finland; rare flora in rare soil. Ann Clin Res 1973; 5: 109-141.

3 de la Chapelle A: Disease gene mapping in isolated human populations: the example of Finland. J M ed Genet 1993; 30: 857-865.

4 Peltonen L, Pekkarinen P, A altonen J: M essages from an isolate: lessons from the Finnish gene pool. Biol Chem 1995; 376: 697-704.

5 Norio R: The diseases of Finland and Scandinavia. In: R othschild HR (ed). Biocultural A spects of Disease. A cademic Press: N ew Y ork, 1981, pp 359-415.

6 White PC, New MI, Dupont B: Congenital adrenal hyperplasia. N Engl J M ed 1987; 316: 1519-1524.

7 Morel Y, Miller WL: Clinical and molecular genetics of congenital adrenal hyperplasia due to 21-hydroxylase deficiency. A dv H um G enet 1991; 20: 1-68.

8 Jääskeläinen J, L evo A, Voutilainen R, Partanen J: Population-wide evaluation of disease manifestation in relation to molecular genotype in steroid 21-hydroxylase (CY P21) deficiency: good correlation in a well-defined population. J Clin Endocrinol Metab 1997; 82: 3293-3297.

9 Pang S, Wallace MA, Hofman L et al: Worldwide experience in newborn screening for classical congenital adrenal hyperplasia due to 21-hydroxylase deficiency. Pediatrics 1988; 81: 866-874.

10 Levo A, Partanen J: Mutation-haplotype analysis of steroid 21-hydroxylase (CY P21) deficiency in Finland. I mplications for the population history of defective alleles. H um G enet 1997; 99: 488-497.

11 Lahermo P, Sajantila A, Sistonen P et al: The genetic relationship between Finns and the Finnish Saami (Lapps): A nalysis of nuclear DNA and mtDNA. Am J H um G enet 1996; 58: 1309-1322.

12 Higashi $Y$, Yoshioka H, Yamane M, Gotoh O, FujiiKuriyama $Y$ : Complete nucleotide sequence of two steroid 21-hydroxylase genes tandemly arranged in human chromosome: a pseudogene and a genuine gene. Proc $\mathrm{N}$ atl A cad Sci USA 1986; 83: 2841-2845.

13 White PC, N ew MI, D upont B: Structure of human steroid 21-hydroxylase genes. Proc Natl A cad Sci USA 1986; 83: 5111-5115.

14 White PC, Tusié-L una M-T, New MI, Speiser PW: Mutations in steroid 21-hydroxylase (CY P21). Hum Mutat 1994; 3: 373-378. 
15 Partanen J, K ere J, Wessberg S, Koskimies S: D etermination of deletion sizes in the M H C-linked complement C 4 and steroid 21-hydroxylase genes by pulsed-field gel electrophoresis. G enomics 1989; 5: 345-349.

16 Partanen J, Koskimies S, Sipilä I, Lipsanen V: Majorhistocompatibility-complex gene markers and R FL $P$ analysis of steroid 21-hydroxylase (CY P21) and complement C4 genes in classical congenital adrenal hyperplasia patients in a single population. A m J H um G enet 1989; 44: 660-670.

17 Campbell RD, Trowsdale J: Map of the human MHC. Immunol Today 1993; 14: 349-352.

18 Sirén MK, Sareneva H, L okki ML, Koskimies S: U nique $\mathrm{HLA}$ antigen frequencies in the Finnish population. Tissue A ntigens 1996; 48: 703-707.

19 L evo A, Westman P, Partanen J : A n approach to mapping haplotype-specific recombination sites in human $\mathrm{MHC}$ class III. I mmunogenetics 1996; 43: 136-140.

20 Laitinen T, Kauppi P, I gnatius J et al: $G$ enetic control of serum IgE levels and asthma: linkage disequilibrium studies in an isolated population. H um M ol G enet 1997; 6: 2069-2076.

21 Kuokkanen S, Sundvall M, Terwilliger J D et al: A putative vulnerability locus to multiple sclerosis maps to 5p14-p12 in a region synthetic to the murine locus $\mathrm{E}$ ae2. $\mathrm{N}$ at $\mathrm{G}$ enet 1996; 13: 477-480.
22 Huurre M: 9000 vuotta Suomen esihistoriaa. 5th edn. K euruu, K ustannusosakeyhtiö O tava, 1995.

23 Jutikkala E: A tlas of Finnish $\mathrm{H}$ istory. 2nd edn, revised. Werner Söderström O y: Porvoo, 1959.

24 Vahtola J: The main phases of colonization in northern Finland. FA R A VID, A cta Soc H ist Finland Septent 1993; 16: 141-151.

25 Sinnot PJ, Costigan C, D yer PA, Harris R, Strachan T: Extended M H C haplotypes and CY P21/C 4 gene organisation in Irish 21-hydroxylase deficiency families. Hum Genet 1991; 87: 361-366.

26 Huurre M, Virrankoski P, Vilkuna K: Suur-Kalajoen historia I. Keski-Pohjanmaan Kirjapaino Oy: Kokkola, 1956.

27 Kere J, E stivill X, Chillón M et al: Cystic fibrosis in a lowincidence population: two major mutations in Finland. H um G enet 1994; 93: 162-166.

28 I käheimo I, Silvennoinen-K assinen S, Tiilikainen A : H LA five-locus haplotypes in Finns. E ur J I mmunogenet 1996; 23: 321-328.

29 Thomsen $M$, B armada $M$, B ois $M$ et al. M H C recombinant families. In: Charron D (ed). G enetic diversity of $H \mathrm{LA}$ Functional and Medical Implication. Proceedings of the Twelfth International $\mathrm{H}$ istocompatibility Workshop and Conference. EDK Medical and Scientific International Publisher: Paris 1997, vol 1, pp 188-195. 